\title{
PRODUCCIÓN MECANIZADA DE MAÍZ, CAMOTE Y YUCA EN LA COSTA DESÉRTICA PERUANA: ESTIMACIÓN DE LA HUELLA DE CARBONO Y PROPUESTAS DE MITIGACIÓN
}

\section{MECHANIZED PRODUCTION OF MAIZE, SWEET POTATO AND CASSAVA IN THE PERUVIAN DESERT COAST: ESTIMATION OF THE CARBON FOOTPRINT AND MITIGATION PROPOSALS}

\author{
Rodrigo A. Morales A ${ }^{1}$, Percy Zorogastúa C. ${ }^{2}$, Felipe de Mendiburu ${ }^{3}$ y Roberto Quiroz ${ }^{4}$
}

\begin{abstract}
Resumen
El objetivo del estudio fue estimar la huella de carbono (HC) en la producción mecanizada de maíz, camote y yuca, así como identificar y proponer opciones de mitigación en la costa del Perú. Se realizó una encuesta a 100 productores de Barranca y Cañete en la región de Lima. Se registraron todas las prácticas de producción y protección de cultivos. La estimación se llevó a cabo con el modelo de simulación programado en Excel; Cool Farm Tool (CFT). El maíz en Barranca registró la mayor cantidad de labranza y aplicaciones de pesticidas. En ambos lugares, se aplicaron dosis medias a altas de $\mathrm{N}$ al maíz, mientras que el camote y yuca sólo recibieron dosis altas. Cuando las emisiones de $\mathrm{CO}_{2}$ por transporte fueron incluidas en los análisis, las fincas que cultivaban yuca en Barranca generaron $267 \mathrm{~kg} \mathrm{CO} 2 \mathrm{eq} \cdot \mathrm{t}^{-1}$, que fue la mayor $\mathrm{HC}\left(132 \mathrm{~kg} \mathrm{CO} 2 \mathrm{eq} \cdot \mathrm{t}^{-1}\right.$ adicionales). Esta adición fue similar en todos los cultivos. Las principales fuentes de emisión de gases de efecto invernadero (GEI) para los tres cultivos fueron la aplicación (49\%) y la producción (38\%) de fertilizantes, seguida del uso de plaguicidas (13\%). Las opciones de mitigación simuladas con el CCAFS-Mitigation Options Tools (CCAFS-MOT) incluyeron el uso de labranza mínima, la incorporación de residuos agrícolas, el uso de fertilizantes orgánicos procesados e inhibidores de nitrificación. Al incorporar las cuatro opciones de mitigación se estimaron reducciones de GEI en $42 \%, 22 \%$ y $26 \%$ para el maíz, camote y la yuca, respectivamente.
\end{abstract}

Palabras clave: $\mathrm{CO}_{2}, \mathrm{CH}_{4}, \mathrm{~N}_{2} \mathrm{O}$, gasto energético, fertilizantes, pesticidas, Barranca, Cañete.

\begin{abstract}
The objective of the study was to estimate the carbon footprint (CFP) in the mechanized production of maize, sweet potato and cassava, and to identify and propose mitigation options in the coast of Peru. A survey was conducted in 100 producers from Barranca and Cañete in the Lima region. All production and crop protection practices were registered. The estimation was then conducted with the Excel-based in simulation model; Cool Farm Tool (CFT). Maize in Barranca registered the highest quantity of tillage and applications of pesticides. In both locations, middle to high doses of $\mathrm{N}$ were applied to maize whereas sweet potato and cassava received only high doses. When $\mathrm{CO}_{2}$ emissions due to transportation were included in the analyses, farms cultivating cassava in Barranca generated the largest CFP of $267 \mathrm{~kg} \mathrm{CO} \mathrm{eq}^{-\mathrm{t}^{-1}}$ (adding $132 \mathrm{~kg} \mathrm{CO} \mathrm{eq}^{-\mathrm{t}^{-1}}$ ). This addition was similar for all crops. The main sources of greenhouse gases emission (GHE) for all three crops were application (49\%), and production (38\%) of fertilizers, followed by the use of pesticides (13\%). Mitigation options simulated with the CCAFS-MOT included the use of minimum tillage, incorporation of agricultural residues, use of processed organic fertilizers and nitrification inhibitors. By incorporating the four mitigation options GHG reductions in $42 \%, 22 \%$ and $26 \%$ were estimated for maize, sweet potato and cassava, respectively.
\end{abstract}

Key words: $\mathrm{CO}_{2}, \mathrm{CH}_{4}, \mathrm{~N}_{2} \mathrm{O}$, energy costs, fertilizers, pesticides, Barranca, Cañete.

\section{Introducción.}

Los cultivos de maíz (Zea mays L.), camote [(Ipomoea batatas (L.) Lam.] y yuca (Manihot esculenta, Crantz), están considerados entre los cultivos tropicales más consumidos a nivel mundial (Montaldo, 1991; Bolaños, 1997; FAO, 2015). En Perú, el Ministerio de Agricultura y Riego (MINAGRI, 2015), reportó que el maíz es el segundo cultivo más sembrado con 326532 ha, con tendencia al incremento en el tiempo. La yuca es el séptimo cultivo de mayor área de siembra con 123197 ha y el camote registró siembras de 15704 ha (MINAGRI, 2015). 
Las tendencias actuales de intensificación y comercialización, adoptadas por los agricultores y empresarios, han incrementado el uso de combustibles fósiles (Haverkort \& Hillier, 2011). El alto uso de agroquímicos incrementa los rendimientos y la contaminación ambiental a través de la emisión de gases de efecto invernadero (GEI); conocido como la huella de carbono (HC) (Colomb et al., 2012). Para el IPCC (2013), entre los principales GEI están el $\mathrm{CH}_{4}$, $\mathrm{N}_{2} \mathrm{O}$ y el $\mathrm{CO}_{2}$, que constituyen importantes factores de cambio climático (CC). También estandarizó la unidad equivalente de $\mathrm{CO}_{2}\left(\mathrm{CO}_{2} \mathrm{eq}\right)$, considerando todas las emisiones por su potencial de calentamiento global. Las emisiones se estiman en GEI total emitidas $\left(\mathrm{HC}_{\mathrm{ha}}\right)$ en $\mathrm{kgCO}_{2} \mathrm{eq} \cdot \mathrm{ha}^{-1}$ y la intensidad $\left(\mathrm{HC}_{\mathrm{i}}\right)$ en $\mathrm{kg} \mathrm{CO} \mathrm{CO}_{2} \mathrm{eq} \cdot \mathrm{t}^{-1}$ (Lal, 2004). El World Resources Institute (2014), informó que las emisiones del sector agropecuario del mundo, en 2013, fueron de $5038.69 \mathrm{Mt} \mathrm{CO}_{2} \mathrm{eq}$, de las cuales en Perú fueron de $23.28 \mathrm{Mt} \mathrm{CO}_{2}$ eq (de éstas el $14.71 \%$ son del sector agropecuario). El SEEG Perú (acrónimo del español - Sistema de Estimación de Emisiones de Gases Efecto Invernadero, 2014), indicó que las emisiones de GEI, en el sector agrícola nacional, entre 2000 y 2013, -generado por la fermentación entérica, manejo del estiércol, cultivo de arroz, quema de pastizales y residuos agrícolas y uso de suelos agrícolas- variaron de 23614 a $27006 \mathrm{Gg} \mathrm{CO}_{2} \mathrm{eq}$ (incremento del 12.56\%). De éstos, el $16 \%$ corresponde a cultivos, $74.1 \%$ a la ganadería y $9.9 \%$ a otras actividades agrícolas.

La estimación de la $\mathrm{HC}$ en la agricultura es importante para analizar su contribución potencial al CC (Haverkort \& Hillier, 2011). Para su cuantificación se utilizan modelos basados en procesos empíricos de las mediciones directas del manejo de la tierra; así como inventarios nacionales realizados por organismos especializados (Olander, 2011). Sin embargo, están limitados por la disponibilidad de datos de pequeña escala y las estimaciones difieren con las mediciones locales (Richards et al., 2016). Frohmann \& Olmos (2005), alertaron que la ausencia de estimaciones de $\mathrm{HC}$ son barreras ambientales en las exportaciones hacia los mercados de los países industrializados. Anunciaron la implementación del etiquetado en los productos exportados con información de las emisiones de $\mathrm{CO}_{2}$ en el proceso de producción y transporte. Se han desarrollado herramientas para calcular la HC por efecto de las prácticas agrícolas y forestales (Haverkort \& Hillier, 2011; Colomb et al., 2012). De éstas, el modelo matemático Cool Farm Tool v. 2 - beta 3 (CFT) -programado en el Excel- es fácil de utilizar y está disponible en forma gratuita en https://www.coolfarmtool.org/CftExcel. Este modelo adopta procesos de armonización de datos, calibración y validación (más de 1000 mediciones de emisiones en campos agrícolas), requeridos por los estándares internacionales (Audsley et al., 2009). Su principal limitante es que no dispone de opciones de mitigación.
El modelo CCAFS-Mitigation Options Tools (CCAFSMOT), modela la reducción de $\mathrm{HC}$ por el uso de medidas de mitigación (Smith et al., 2007; Feliciano et al., 2015). Por todo lo expuesto los objetivos planteados en este estudio fueron: 1) estimar la huella de carbono en la producción mecanizada de maíz, camote y yuca, y 2) identificar y proponer opciones de mitigación en la costa del Perú.

\section{Materiales y métodos.}

Encuestas a productores de Barranca y Cañete.

Durante el periodo noviembre de 2015 a enero de 2016, se realizaron 100 encuestas a productores de maíz (36), camote (30) y yuca (34), con error de la muestra de $9.8 \%$ de una población finita (corresponde al error en la estimación del valor que ingresa a los modelos y de la estimación de GEI y las medidas de mitigación) (Cochran, 2000). En la provincia de Barranca se encuestaron a productores de La Campiña de Supe, Arguay, San José de Pativilca y el Centro Poblado Santa Elena Norte y Sur. En Cañete, en Hualcará, Herbay y el Centro Poblado Menor de Unanue. Las entrevistas se usaron para generar los insumos para que los modelos estimaran las emisiones de GEI. Por lo tanto; las encuestas consistieron en preguntas específicas para cuantificar las emisiones por las prácticas de producción. Incluyen la preparación de suelos, las aplicaciones de agroquímicos en general, rendimientos comerciales y el transporte de cosechas. La información obtenida se verificó con los técnicos expertos de los tres cultivos y comprobada con la base de datos oficiales del MINAGRI (2015).

Descripción de localidades y variedades sembradas.

Los suelos de las localidades estudiadas, comprenden el Regosol, Leptosol y Fluvisol (Gardi et al., 2014). Debido a la escasa precipitación, se implementaron módulos de riego de 5000 a 7000 $\mathrm{m}^{3} \cdot \mathrm{ha}^{-1}$, pertenecientes a la junta de usuarios del sub distrito de riego de Cañete y del distrito de Barranca. La ubicación geoespacial de las fincas se realizó con un GPS (latitud de $10^{\circ}$ a $13^{\circ} \mathrm{S}$ y longitud de $76^{\circ}$ a $77^{\circ} \mathrm{W}$ ), verificada con Google Earth y se utilizó para calcular las distancias de entrega de la cosecha hasta las empresas agroindustriales y al gran mercado central de Lima. Por localidad se seleccionaron fincas, a las cuales se les realizó el análisis físico-químico de suelos en el laboratorio de análisis de suelos, plantas, aguas y fertilizantes de la Universidad Nacional Agraria La Molina, Perú. En Barranca los suelos son de textura Franco arenosa, Arena y Arena Limosa y en Cañete, de textura Arena y Franco (Tabla 1). El pH es básico, con bajos contenidos de nitrógeno y materia orgánica. Los registros de la temperatura ambiental máxima y mínima, precipitación pluvial y humedad relativa (periodo 2005-2015), se obtuvieron de las estaciones meteorológicas de la Universidad Nacional Agraria La Molina (Estación Von Humboldt), de Barranca y San Vicente de Cañete; pertenecientes a la red nacional de 
Tabla 1. Caracterización de suelos y temperatura promedio anual de las localidades de Barranca y Cañete. Perú.

\begin{tabular}{|c|c|c|c|c|c|c|c|}
\hline Localidad & Textura & $\begin{array}{c}\text { C Orgánico } \\
(\%)\end{array}$ & $\begin{array}{c}\mathrm{N} \\
(\%)\end{array}$ & $\begin{array}{l}\mathrm{MO} \\
(\%)\end{array}$ & $\mathrm{pH}$ & $\begin{array}{c}\text { Densidad } \\
\text { Aparente } \\
\left(\mathrm{g} \cdot \mathrm{cm}^{-3}\right)\end{array}$ & $\begin{array}{c}\mathrm{T}^{\circ} \\
\text { promedio anual } \\
\left({ }^{\circ} \mathrm{C}\right)\end{array}$ \\
\hline \multicolumn{8}{|l|}{ Barranca } \\
\hline La Campiña & Franco arenoso & 0.86 & 0.08 & 1.48 & 7.70 & 1.46 & 19.00 \\
\hline Santa Elena Norte & Arena & 0.41 & 0.05 & 0.70 & 8.02 & 1.44 & 18.80 \\
\hline Santa Elena Sur & Arena & 0.23 & 0.04 & 0.47 & 7.85 & 1.49 & 18.51 \\
\hline San José Pativilca & Franco arenoso & 0.64 & 0.06 & 1.04 & 7.09 & 1.31 & 18.51 \\
\hline Arguay & Arena limosa & 1.05 & 1.00 & 1.81 & 7.00 & 1.42 & 18.51 \\
\hline \multicolumn{8}{|l|}{ Cañete } \\
\hline Hualcará & Arena & 0.17 & 0.03 & 0.29 & 7.54 & 1.50 & 24.33 \\
\hline Unanue & Arena & 0.09 & 0.03 & 0.16 & 7.20 & 1.49 & 21.50 \\
\hline Herbay & Franco & 1.14 & 0.08 & 2.14 & 7.78 & 1.42 & 21.50 \\
\hline
\end{tabular}

Fuente: Laboratorio de Análisis de Suelos, Plantas, Aguas y Fertilizantes de la UNALM. C Orgánico: Carbono Orgánico, MO: Materia Orgánica.

estaciones meteorológicas del Servicio Nacional de Meteorología e Hidrología del Perú (SENAMHI).

En el cultivo de maíz, los productores siembran los híbridos P30F35, D8008, D5005, D7088, BG-9621 у XB-8010. Los camotes de pulpa naranja sembrados son de las variedades Jonathan, Huambachero INIA y Jewel, los de pulpa morada se siembran las variedades Morado Milagroso y Morado Limeño. En el cultivo de yuca las principales variedades sembradas son Amarilla Criolla y Señorita.

Descripción del modelo de simulación CFT.

Dado que los modelos son, por definición, simples representaciones de los procesos reales, las estimaciones se basan en suposiciones basadas en la ciencia sobre las interacciones dinámicas entre el suelo y su manejo, el clima y otros componentes (Wolf, 2002; Rosenzweig et al., 2013). El modelo CFT está conformado de sub modelos de estimación -p.e. de máxima verosimilitud restringida- de emisiones globales de $\mathrm{GEI}\left(\mathrm{CO}_{2}, \mathrm{~N}_{2} \mathrm{O}\right.$ y $\mathrm{NO}$ por la producción y aplicación de urea y fertilizantes, plaguicidas y labores de labranza). Para ello, el modelo CFT incorporó la siguiente ecuación (Bouwman et al., 2002):

$$
\log (\text { Nemission })=A+\sum_{i=1}^{n} E i
$$

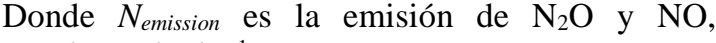
expresado en $\mathrm{kg} \cdot \mathrm{ha}^{-1}$ de $\mathrm{N}$, durante el período de las medidas, $A$ es una constante y $E i$ es el valor para el factor de efecto $i$. Los factores de control (factor $i$ ), para las emisiones de $\mathrm{N}_{2} \mathrm{O}$ y $\mathrm{NO}$ de los campos agrícolas, por sistemas de producción, fueron la tasa de aplicación de $\mathrm{N}, \mathrm{pH}$ del suelo, textura del suelo, el clima y el tipo de cultivo.

El modelo CFT, integra los factores de emisión para la producción de fertilizantes en Europa y del promedio mundial para la producción de fertilizantes, complementadas con información de la energía utilizada para extraer la materia prima, así como las emisiones asociadas con el transporte de la materia prima a la planta de procesamiento (FAO-IFA, 2004; IFA, 2009; Zhang et al., 2013). Considera las emisiones de las prácticas agronómicas, protección de cultivos, el uso directo de energía para las labores agrícolas y el transporte de productos de cosechas de cultivos y de la actividad ganadera (Ogle et al., 2005; ASABE, 2006; Audsley et al., 2009). Para la estimación de las emisiones de GEI por la incorporación de residuos, CFT utiliza la siguiente ecuación (IPCC, 2007):

$$
\mathrm{N}_{2} \mathrm{O}-\mathrm{N}_{\text {Ninputs }}=\mathrm{F}_{C R} \times E F_{1}
$$

Donde $F_{C R}$ es la cantidad de $\mathrm{N}$ en residuos de cultivos -en la superficie y dentro del suelo- $\left(\mathrm{kg} \cdot \mathrm{N} \cdot \mathrm{anno}^{-}\right.$ ${ }^{1}$ ), y $E F_{1}$ es el factor de emisión de $\mathrm{N}_{2} \mathrm{O}$ debido a las adiciones de $\mathrm{N}$ de los residuos de los cultivos $\left[\mathrm{kg} \mathrm{N}_{2} \mathrm{O}\right.$ $\mathrm{N}(\mathrm{kg} \mathrm{N})^{-1}$ ]. El valor predeterminado para $E F_{1}$ es 0.01 con rango de incertidumbre de 0.003 a 0.03 (IPCC, 2007). Para la estimación de las emisiones de $\mathrm{CH}_{4}(\mathrm{~g})$ y $\mathrm{N}_{2} \mathrm{O}$ (g), debido a la quema de los residuos de los cultivos, incorpora la ecuación del IPCC (2013): Emisssion $=A \times E F$. Donde $A$ es la cantidad total de biomasa quemada ( $\mathrm{kg}$ de materia seca) y $E F$ es el factor de emisión del IPCC (en $\mathrm{CH}_{4}$ y $\mathrm{N}_{2} \mathrm{O} / \mathrm{kg}$ de materia seca). Los valores de $E F$, por defecto, fueron 0.01 (IPCC, 2007).

Richards et al. (2016), confirmaron la validez de las estimaciones de $\mathrm{HC}$ en cultivos básicos en todas las fincas dependientes de grandes cantidades de insumos, por medio del modelo CFT en zonas desérticas y clima templado. A su vez, sugieren la obtención de meta datos en climas tropicales. Coinciden con Milne et al. (2013), los cuales señalaron que para reducir las incertidumbres en la estimación de GEI, se necesitan estudios cuantitativos en diversos tipos y prácticas de manejo de suelos por región climatológica. Para el uso del modelo CFT, se seleccionaron las siguientes hojas de trabajo de las nueve existentes: 1. Información general (país, localidad, año, producto, rendimiento por área, clima y temperatura promedio anual); 2. Manejo de cultivos (cultivo, suelos, producto, nutrientes, cantidad y método de aplicaciones de fertilizantes y el número de aplicaciones de plaguicidas). Cada dosis de ingrediente activo por plaguicida se cuenta como una aplicación, ya sea aplicado solo o en mezclas; 5. Uso de energía en campo (electricidad y combustibles por equipos desde 
Tabla 2. Promedio de actividades agronómicas, aplicaciones de fitosanitarios y rendimientos comerciales de fincas de Barranca y Cañete productoras de maíz, camote y yuca, y rendimiento comercial de la costa peruana.

\begin{tabular}{lcccccc}
\hline \multirow{2}{*}{ Descripción } & \multicolumn{3}{c}{ Barranca } & \multicolumn{3}{c}{ Cañete } \\
\cline { 2 - 7 } & Maíz & Camote & Yuca & Maíz & Camote & Yuca \\
\hline Operaciones de labranza & 8 & 10 & 8 & 8 & 5 & 8 \\
Aplicaciones de plaguicidas & 14 & 15 & 10 & 10 & 10 & 10 \\
Siembra & mecanizada* $^{*}$ manual & manual & mecanizada & manual & manual \\
Cosecha & mecanizada* & manual & manual & manual & manual & manual \\
Rendimiento $\left(\mathrm{t} \cdot \mathrm{ha}^{-1}\right.$ ) & 11.5 & 25 & 18 & 10.75 & 26.25 & 25.75 \\
Rendimiento de costa $\left(\mathrm{t} \cdot \mathrm{ha}^{-1}\right)^{* *}$ & 7.32 & 17.75 & 17.69 & & & \\
\hline$* 50 \%$ de prod
\end{tabular}

* 50\% de productores. ** Fuente: MINAGRI (2015). Rendimiento de granos de maíz a 14\% humedad y frescos

de raíces de almacenamiento de camote y yuca.

la preparación de suelos hasta la cosecha); y 7. Transporte de cosechas. En Perú, las emisiones por el transporte, son asignados a la agroindustria y los compradores. Esto permitió hacer una comparación entre las $\mathrm{HC}$ a nivel de fincas. Pero, en muchas ocasiones les corresponde a los productores cubrir el costo de transporte de cosechas. Para el cálculo de la HC total por cultivo y por provincias estudiadas, se procedió a la sumatoria de las emisiones por la producción y aplicación de fertilizantes, por el uso de plaguicidas, el uso energético de cultivos a nivel de fincas y por el transporte de cosechas.

Estimación del potencial de mitigación con el modelo CCAFS-MOT.

El modelo CCAFS-MOT incorporó 16 prácticas de mitigación, las cuales pueden reducir o evitar las emisiones de GEI de tierras agrícolas (Moran et al., 2008; Akiyama et al., 2010; Linquist et al., 2012; Shan \& Yan, 2013; van Kessel et al., 2013; Benites \& Bot, 2014; Nayak et al., 2015). Para el modelamiento de las prácticas de mitigación, se seleccionaron las hojas de entrada general (con información de región, suelos, cambios en el uso de tierra y zona ecológica), cultivos (tipo de cultivo, rendimientos por unidad agrícola, promedio de región, manejo de suelos, el tipo y cantidad de fertilizantes) y mitigación. Las opciones individuales de prácticas de mitigación modeladas por cultivo fueron consultadas a los agricultores, obteniéndose escenarios potenciales de reducción de las emisiones de GEI.

\section{Resultados y discusión.}

Componentes agronómicos, fitosanitarios y rendimientos.

Las provincias de Barranca y Cañete representan al sistema de producción de los cultivos estudiados en las costas central y norte de Perú. En estas zonas se siembra el $48.2 \%$ del área total de maíz, $72 \%$ de camote y $5 \%$ de yuca (MINAGRI, 2015). Los productores de maíz señalaron que se presentan las plagas Agrotis spp., [Spodoptera frugiperda (J. E. Smith)], Heliothis zea (Boddie), áfidos y la enfermedad mancha de asfalto causada por la interacción Phyllachora maydis, Monographella maydis y Coniothyrium phyllachorae. En Barranca realizan 14 aplicaciones de plaguicidas para el control. El rendimiento promedio reportado fue de $11.5 \mathrm{t} \cdot \mathrm{ha}^{-1}$, donde el $50 \%$ de los productores realizan la siembra y cosecha mecanizada. En las fincas de Barranca y Cañete realizan en promedio ocho operaciones de labranza convencional. Se realizan 10 aplicaciones de plaguicidas y el rendimiento promedio es de $10.75 \mathrm{t} \cdot \mathrm{ha}^{-1}$ (Tabla 2). La cosecha es manual y para el secado de las mazorcas se utiliza la radiación solar. El desgrane se efectúa al 14\% de humedad en los granos (verificado por la agroindustria). El peso fresco comercial de camote y yuca son estimados en conjunto con los compradores.

En las plantas de camote se presentan las plagas Agrotis spp., Prodenia (=Spodoptera) eridania (Cramer), áfidos y enfermedades bacterianas. En Barranca realizan 10 operaciones de labranza y 15 aplicaciones de plaguicidas; cuyo rendimiento promedio fue de $25 \mathrm{t} \cdot \mathrm{ha}^{-1}$. En Cañete realizan cinco operaciones de labranza, con 10 aplicaciones de plaguicidas y el rendimiento promedio es de $26.25 \mathrm{t} \cdot \mathrm{ha}^{-}$ 1. Indicaron que el follaje fresco es utilizado para la alimentación de animales de especies menores y ganado vacuno. En el cultivo de yuca en Barranca y Cañete se llevan a cabo ocho labores de labranza y se realizan en promedio 10 aplicaciones de plaguicidas para el control de Erinnyis ello (L.), Tetranychus sp., Trialeurodes spp. y Oidium spp. En Barranca los rendimientos de yuca fueron de $18 \mathrm{t} \cdot \mathrm{ha}^{-1} \mathrm{y}$ en Cañete de $25.75 \mathrm{t} \cdot \mathrm{ha}^{-1}$. En la Tabla 2, se muestra que los rendimientos reportados en los tres cultivos en los dos sitios, superaron los rendimientos promedios de la costa peruana - periodo 2010 a 2014- de 7.32, 17.75 y 17.69 $\mathrm{t} \cdot \mathrm{ha}^{-1}$; respectivamente (MINAGRI, 2015).

Los altos rendimientos pueden atribuirse a las altas densidades de siembra (en maíz hasta 9 plantas $\cdot \mathrm{m}^{2}$, en camote hasta 7.4 plantas $\cdot \mathrm{m}^{2}$ y en yuca 1 planta $\cdot \mathrm{m}^{2}$ ), al uso de cultivares con alto potencial de rendimientos adaptados al clima desértico, uso de riego, control de plagas y enfermedades y los niveles de fertilización. Según Pandey \& Gardner (1992), los altos rendimientos en maíz es reflejo del uso de híbridos con tolerancia a altas densidades $\mathrm{y}$ a las plagas $\mathrm{y}$ enfermedades.

Todos los agricultores encuestados aplicaron urea a los tres cultivos (Tabla 3 ). Otras fuentes variaron con el 
Tabla 3. Niveles de N, P y K $\left(\mathrm{kg} \cdot \mathrm{ha}^{-1}\right)$ y fertilizantes aplicados en los cultivos de maíz, camote y yuca en Barranca y Cañete. Perú.

\begin{tabular}{|c|c|c|c|c|c|c|c|c|c|c|c|c|c|c|}
\hline \multirow{2}{*}{ Localidad } & \multicolumn{5}{|c|}{ Maíz } & \multicolumn{5}{|c|}{ Camote } & \multicolumn{4}{|c|}{ Yuca } \\
\hline & $\mathrm{N}$ & $\mathrm{P}$ & $\mathrm{K}$ & & Fertilizantes & $\mathrm{N}$ & $\mathrm{P}$ & $\mathrm{K}$ & & Fertilizantes & $\mathrm{N}$ & $\mathrm{P}$ & $\mathrm{K}$ & Fertilizantes \\
\hline Barranca & $\begin{array}{l}119 \quad \mathrm{a} \\
345\end{array}$ & $\begin{array}{l}69 \text { a } \\
92\end{array}$ & $\begin{array}{l}33 \\
75\end{array}$ & a & $\begin{array}{l}\text { Urea } \\
\mathrm{NH}_{4} \mathrm{NO}_{3} \\
0-46-0 \\
\left(\mathrm{NH}_{4}\right)_{2} \mathrm{HPO}_{4} \\
\mathrm{KCl} \\
\end{array}$ & $\begin{array}{l}80 \quad a \\
128\end{array}$ & $\begin{array}{l}60 \mathrm{a} \\
92\end{array}$ & $\begin{array}{l}33 \\
50\end{array}$ & $\mathrm{a}$ & $\begin{array}{l}\text { Urea } \\
\mathrm{NH}_{4} \mathrm{NO}_{3} \\
\left(\mathrm{NH}_{4}\right)_{2} \mathrm{HPO}_{4} \\
\mathrm{KCl} \\
\mathrm{K}_{2} \mathrm{SO}_{4} \\
\end{array}$ & $\begin{array}{l}174 \mathrm{a} \\
300\end{array}$ & $\begin{array}{l}81 \text { a } \\
92\end{array}$ & $\begin{array}{l}50 \mathrm{a} \\
68\end{array}$ & $\begin{array}{l}\text { Urea } \\
\left(\mathrm{NH}_{4}\right)_{2} \mathrm{HPO}_{4} \\
0-46-0 \\
\mathrm{KCl}\end{array}$ \\
\hline Cañete & $\begin{array}{l}105 \quad \mathrm{a} \\
200\end{array}$ & $\begin{array}{l}40 \mathrm{a} \\
120\end{array}$ & $\begin{array}{l}100 \\
140\end{array}$ & a & $\begin{array}{l}\text { Urea } \\
0-46-0 \\
20-20-20 \\
\mathrm{KCl}\end{array}$ & $\begin{array}{l}92 \mathrm{a} \\
112\end{array}$ & 92 & $\begin{array}{l}50 \\
100\end{array}$ & $\mathrm{a}$ & $\begin{array}{l}\text { Urea } \\
\mathrm{NH}_{4} \mathrm{NO}_{3} \\
0-46-0 \\
\left(\mathrm{NH}_{4}\right)_{2} \mathrm{HPO}_{4} \\
\mathrm{KCl} \\
\mathrm{Ca}\left(\mathrm{H}_{2} \mathrm{PO}_{4}\right)_{2}\end{array}$ & $\begin{array}{l}92 \mathrm{a} \\
132\end{array}$ & $\begin{array}{l}40 \mathrm{a} \\
92\end{array}$ & $\begin{array}{l}50 \mathrm{a} \\
140\end{array}$ & $\begin{array}{l}\text { Urea } \\
20-20-20 \\
0-46-0 \\
\left(\mathrm{NH}_{4}\right)_{2} \mathrm{HPO}_{4} \\
\mathrm{KCl}\end{array}$ \\
\hline
\end{tabular}

cultivo y el sitio. Los productores de maíz de ambos sitios informaron usar $\mathrm{NH}_{4} \mathrm{NO}_{3},\left(\mathrm{NH}_{4}\right)_{2} \mathrm{HPO}_{4}$ y $20-20$ 20. Los productores de camote en Barranca fertilizaron con $\mathrm{NH}_{4} \mathrm{NO}_{3},\left(\mathrm{NH}_{4}\right)_{2} \mathrm{HPO}_{4}$; el $\mathrm{KCl}$ y $\mathrm{K}_{2} \mathrm{SO}_{4}$ como fuentes de $\mathrm{K}_{2} \mathrm{O}$. Mientras que en Cañete aplicaron $\mathrm{NH}_{4} \mathrm{NO}_{3}, \quad\left(\mathrm{NH}_{4}\right)_{2} \mathrm{HPO}_{4}, \mathrm{Ca}\left(\mathrm{H}_{2} \mathrm{PO}_{4}\right)_{2}$ y 0-46-0. Los productores de yuca en Barranca aplicaron $\left(\mathrm{NH}_{4}\right)_{2} \mathrm{HPO}_{4}$ y 0-46-0. En Cañete, se aplicaron $\left(\mathrm{NH}_{4}\right)_{2} \mathrm{HPO}_{4}, 20-20-20$ y 0-46-0. En el maíz, en ambos sitios, se aplicaron dosis medias a altas de $\mathrm{N}$; se aplicaron dosis altas de $\mathrm{N}$ en el camote y yuca. En maíz, se informó de aplicaciones de dosis medias y altas de $\mathrm{P}$ y K, y medias en camote y yuca.

Estimación de las emisiones de GEI en fincas de maíz, camote y yuca.

Emisiones por la producción y aplicación de fertilizantes.

En las fincas de maíz en Barranca, por la producción de fertilizantes, se estimaron altas emisiones promedio de $1278 \mathrm{~kg} \mathrm{CO}$ eq.ha ${ }^{-1}$ (Tabla 4). Le siguieron las emisiones en camote de Cañete y de yuca en Barranca con promedios de $963 \mathrm{~kg}$ y $712 \mathrm{~kg} \mathrm{CO} \mathrm{CO}_{2} \mathrm{eq} \cdot \mathrm{ha}^{-1}$; respectivamente. Por la aplicación de fertilizantes, las mayores emisiones se calcularon en Barranca en las fincas de yuca y maíz con promedio de $892 \mathrm{~kg} \mathrm{CO}$ eq $\cdot \mathrm{ha}^{-1}$ y $574 \mathrm{NO}_{2}(\mathrm{~kg} \mathrm{CO}$ eq.ha-1) y de $589 \mathrm{~kg} \mathrm{CO}$ eq $\cdot \mathrm{ha}^{-1}$ y $403 \mathrm{NO}_{2}\left(\mathrm{~kg} \mathrm{CO}\right.$ eq $\left.\cdot \mathrm{ha}^{-1}\right)$; respectivamente. Se desprende que la producción de los fertilizantes requiere del uso de gran cantidad de combustibles fósiles, es dependiente de las tecnologías de producción y del país donde se fabrica el fertilizante (Brentrup \& Pallière, 2008). Las estadísticas del MINAGRI (2015), registran que el $98 \%$ de las importaciones de fertilizantes nitrogenados provienen de Estados Unidos.

La aplicación de estos fertilizantes aumenta las emisiones de $\mathrm{N}_{2} \mathrm{O}$ y $\mathrm{NO}$ y $\mathrm{NH}_{3}$, por medio de la nitrificación, desnitrificación y volatización que se produce naturalmente en los suelos (IPCC, 2013). Las fincas de maíz en Barranca, caracterizadas por las aplicaciones de altos niveles de $\mathrm{N}$ (hasta $345 \mathrm{~kg}$ ) y con los mayores rendimientos, influyeron en la $\mathrm{HC}_{\mathrm{ha}}$ promedio calculada de $2578 \mathrm{~kg} \mathrm{CO} 2 \mathrm{eq} \cdot \mathrm{ha}^{-1}$. Esta $\mathrm{HC}_{\mathrm{ha}}$ reflejó el alto y continuo uso de agroquímicos, especialmente la fertilización nitrogenada. $\mathrm{La} \mathrm{HC}_{\mathrm{i}}$ promedio resultante $\left(224 \mathrm{~kg} \mathrm{CO} \mathrm{CO}_{2} \cdot \mathrm{t}^{-1}\right)$, es menor al reporte de maíz en La Pampa, Argentina con $300 \mathrm{~kg}$ $\mathrm{CO}_{2} \mathrm{eq} \cdot \mathrm{t}^{-1}$ (Frank et al., 2014). También son coherentes con las HC obtenidas en campos sembrados con maíz en Canadá por 19 años continuos (Ma et al., 2014). En Perú, la producción nacional de maíz no cubre la demanda de 4 millones de toneladas requeridas por el sector avícola que es el mayor consumidor (FAO, 2015). Por lo tanto, hay programaciones oficiales de aumento del área de siembra concomitante al incremento de altas aplicaciones de N. El maíz es considerado gran extractor de nutrientes del suelo, restituyéndose periódicamente con fertilizantes nitrogenados en su proceso productivo. El sistema de producción de camote y yuca también está basado en

Tabla 4. Emisiones promedio de $\mathrm{CO}_{2}$ por componente de producción en fincas de maíz, camote y yuca, y las respectivas HC estimadas con el modelo CFT. Perú.

\begin{tabular}{|c|c|c|c|c|c|c|}
\hline \multirow{2}{*}{ Componente } & \multicolumn{3}{|c|}{ Barranca } & \multicolumn{3}{|c|}{ Cañete } \\
\hline & Maíz & Camote & Yuca & Maíz & Camote & Yuca \\
\hline Producción de fertilizantes $\left(\mathrm{kg} \mathrm{CO}_{2} \mathrm{eq} \cdot \mathrm{ha}^{-1}\right)$ & 1278 & 517 & 712 & 538 & 963 & 543 \\
\hline Aplicación de fertilizantes $\left(\mathrm{kg} \mathrm{CO} 2 \mathrm{eq} \cdot \mathrm{ha}^{-1}\right)$ & 589 & 464 & 892 & 439 & 544 & 473 \\
\hline $\mathrm{N}_{2} \mathrm{O}\left(\mathrm{kg} \mathrm{CO} 2 \mathrm{eq} \cdot \mathrm{ha}^{-1}\right)$ & 403 & 285 & 574 & 184 & 332 & 319 \\
\hline Uso de plaguicidas $\left(\mathrm{kg} \mathrm{CO} 2 \mathrm{eq} \cdot \mathrm{ha}^{-1}\right)$ & 205 & 294 & 205 & 205 & 205 & 205 \\
\hline Uso energía en campo $\left(\mathrm{kg} \mathrm{CO} 2 \mathrm{eq} \cdot \mathrm{ha}^{-1}\right)$ & 103 & 65 & 55 & 60 & 50 & 59 \\
\hline Sub Total $\left(\mathrm{kg} \mathrm{CO} 2 \mathrm{eq} \cdot \mathrm{t}^{-1}\right)$ & 224 & 65 & 135 & 133 & 80 & 62 \\
\hline Sub Total $\left(\mathrm{kg} \mathrm{CO} 2 \mathrm{eq} \cdot \mathrm{ha}^{-1}\right)$ & 2578 & 1625 & 2438 & 1426 & 2094 & 1599 \\
\hline Transporte cosecha $\left(\mathrm{kg} \mathrm{CO} 2 \mathrm{eq} \cdot \mathrm{ha}^{-1}\right)$ & 185 & 3503 & 2374 & 334 & 2348 & 2287 \\
\hline Total $\left(\mathrm{kg} \mathrm{CO} 2 \mathrm{eq} \cdot \mathrm{t}^{-1}\right)$ & 240 & 205 & 267 & 164 & 169 & 151 \\
\hline Total $\left(\mathrm{kg} \mathrm{CO} 2 \mathrm{eq} \cdot \mathrm{ha}^{-1}\right)$ & 2763 & 5128 & 4812 & 1760 & 4442 & 3886 \\
\hline
\end{tabular}


aplicaciones de altos niveles de N. Esta práctica agrícola concuerda con los reportes de la FAO-IFA (2004), al señalar los incrementos en el uso de fertilizantes nitrogenados, en el tiempo, especialmente en los países en vías de desarrollo.

Estimación de la HC por el uso energético de cultivos a nivel de fincas.

Las emisiones de GEI por el uso de plaguicidas fueron similares en los tres cultivos de los dos sitios (promedio de $205 \mathrm{~kg} \mathrm{CO}$ eq $\cdot \mathrm{ha}^{-1}$ ), a excepción del camote en Barranca con promedio de $294 \mathrm{~kg} \mathrm{CO} 2 \mathrm{eq} \cdot \mathrm{ha}^{-}$

1 , donde se aplica la mayor cantidad de plaguicidas. Se estimó que en las fincas de maíz, camote y yuca de Barranca la energía directa utilizada en las labores agronómicas fue de 1 461, 918 y $779 \mathrm{MJ} \cdot \mathrm{ha}^{-1}$; respectivamente. Chaparro et al. (2005), calcularon que el uso energético para la labranza convencional en maíz del Valle de Cauca, Colombia fue de $3634 \mathrm{MJ} \cdot \mathrm{ha}^{-1}$. El mayor gasto energético en las fincas de maíz en Barranca, se reflejó en la mayor emisión promedio de $103 \mathrm{~kg} \mathrm{CO}$ eq $\cdot \mathrm{ha}^{-1}$.

La menor $\mathrm{HC}_{\text {ha }}$ se obtuvo en las fincas de maíz en Cañete con promedio de $1426 \mathrm{~kg} \mathrm{CO}$ eq $\cdot \mathrm{ha}^{-1}$. Por los altos rendimientos de raíces comerciales de yuca en Cañete y camote en Barranca, se estimaron las menores $\mathrm{HC}_{\mathrm{i}}$ con promedio de 62 y $65 \mathrm{~kg} \mathrm{CO}_{2} \mathrm{eq} \cdot \mathrm{t}^{-1}$; respectivamente. Las $\mathrm{HC}_{\mathrm{i}}$ obtenidas en la producción de camote coinciden con los reportes de Filipinas que varían de 23 a $95 \mathrm{~kg} \mathrm{CO}$ eq.t. $\mathrm{t}^{-1}$ (Flores et al., 2016). Como comparador de las $\mathrm{HC}$ estimadas en la producción de yuca, en Nigeria se reportaron $\mathrm{HC}_{\mathrm{i}}$ de $105 \mathrm{~kg} \mathrm{CO} \mathrm{eq}^{\mathrm{et}} \mathrm{t}^{-1}$ (World Bank, 2013). En las fincas de yuca en Barranca, la $\mathrm{HC}_{\mathrm{i}}$ promedio fue de $135 \mathrm{~kg}$ $\mathrm{CO}_{2} \mathrm{eq} \cdot \mathrm{t}^{-1}$.

HC totales considerando las emisiones por el transporte de cosechas.

En Barranca las cosechas de maíz son transportadas a granel desde las fincas, en vehículos de motor diésel con cargas superiores a 3.5 toneladas, hasta las granjas avícolas en Huaura, con distancia promedio de $24 \mathrm{~km}$. En Cañete son transportados a Chincha (distancia promedio de $50 \mathrm{~km}$ ). Por el transporte de maíz desde Barranca y Cañete las emisiones promedio fueron de 185 y $334 \mathrm{~kg} \mathrm{CO} \mathrm{eq} \cdot \mathrm{ha}^{-1}$; respectivamente. El $90 \%$ de las cosechas de camote y yuca es transportado al gran mercado mayorista de Lima, cuya distancia promedio desde Barranca es de $199 \mathrm{~km}$ y desde Cañete de 166 $\mathrm{km}$. El resto de la cosecha es consumida en los restaurantes locales. Las emisiones de GEI en las fincas de camote y yuca de Barranca, fueron mayores que las de Cañete. En Barranca, también se calcularon las mayores HC totales por cultivo, por el sistema intensivo de producción y por las mayores distancias de transporte de cosechas. El rango de incremento en la $\mathrm{HC}_{\mathrm{i}}$, por el transporte, fue de 16 a $140 \mathrm{~kg} \mathrm{CO} \mathrm{eq}^{-\mathrm{t}^{-1}} \mathrm{en}$ las fincas de maíz y camote en Barranca; respectivamente. Haverkort \& Hillier (2011), indicaron que el transporte incrementó la $\mathrm{HC}_{\mathrm{i}}$ en la producción de papa hasta $12.5 \mathrm{~kg} \mathrm{CO} \mathrm{CO}_{2} \cdot \mathrm{t}^{-1}$. Flores et al. (2016), concluyeron que las $\mathrm{HC}$ difieren según el sistema de utilización de recursos energéticos como el fertilizante químico y el diésel.

En los tres cultivos, el aporte respectivo a las HC por la aplicación y producción de fertilizantes fue de $49 \%$ y $38 \%$, y por el uso de plaguicidas con $13 \%$. De las emisiones por la aplicación de fertilizantes por sitios, en la producción de yuca en Barranca y Cañete aportó el $61 \%$ y $51 \%$; respectivamente. Se confirmó que los fertilizantes en la agricultura son considerados como la fuente antropogénica más importante de emisión de GEI. Además, producen efectos negativos directos en la inocuidad de alimentos, alteran el equilibrio ambiental y con ello, alta contaminación. De las emisiones de GEI del 2013 del sector agropecuario nacional, la HC total calculada en los tres cultivos en el sistema de producción de costa, representó el $1.5 \%$ del total de las emisiones. $\mathrm{La} \mathrm{HC}_{\mathrm{ha}}$ de maíz acumuló $0.87 \%$, de camote el $1.85 \%$ y de yuca el $1.68 \%$. En las estadísticas nacionales oficiales no hay reportes de las $\mathrm{HC}$ de estos tres cultivos; por lo tanto, este estudio adquiere gran importancia. Por lo tanto; se confirmó que el cálculo de la $\mathrm{HC}$ por sistema de cultivo es un aporte valioso para una política eficiente y gestión de la sustentabilidad para afrontar el CC.

Evaluación del impacto potencial de las prácticas de mitigación.

Con el CCAFS-MOT se modelaron diversas opciones de mitigación en la producción de maíz, camote y yuca con el sistema de producción mecanizado, sin afectar la capacidad de producción, con reducción energética proveniente de los combustibles fósiles y, por consiguiente, reducción del impacto ambiental y la conservación de los recursos energéticos no renovables (IPCC, 2007; Andrade et al., 2008; IFA, 2009; Olivet et al., 2012; Herrero et al., 2013). Las prácticas de mitigación modeladas fueron consultadas con los productores para determinar la factibilidad práctica de su implementación con la finalidad de transformar una posible amenaza en una oportunidad (Tabla 5). Los escenarios de producción que se conformaron por cultivo, con las mejores propuestas de mitigación, fueron; i) incorporación de residuos agrícolas para mejorar la fertilidad de suelos (utilizada solo en algunas unidades productivas de Barranca), ii) la aplicación de abonos orgánicos procesados. Posee un nivel de aplicabilidad media. Implica bajos costos adicionales ya que su valor es bajo $\mathrm{y}$ es transportado en los mismos vehículos que transporta las cosechas. Técnicamente se debe contemplar que los suelos con alta humedad se tornan en emisores de $\mathrm{CH}_{4}$ y con poca humedad en sumideros (Nyakatawa et al., 2011), iii) la labranza mínima que consiste en un solo pase de rastra semi pesada para la preparación de terreno, y iv) uso de inhibidores de nitrificación, los cuales evitan que el $\mathrm{NH}_{4}$ se transforme en $\mathrm{NO}_{2}$ y finalmente en $\mathrm{NO}_{3}$ (Akiyama et al., 2010). Se 
Tabla 5. Opciones de estrategias de mitigación de $\mathrm{HC}$ en la producción mecanizada de maíz, camote y yuca. Barranca y Cañete. Perú.

\begin{tabular}{lccccc}
\hline \multirow{2}{*}{ Cultivo } & \multicolumn{2}{c}{ Prácticas de mitigación (reducción promedio $\mathrm{kg} \mathrm{CO}_{2} \mathrm{eq} \cdot \mathrm{ha}^{-1}$ ) } \\
\cline { 2 - 6 } & $\begin{array}{c}\text { HCha de } \\
\text { referencia } \\
\left.\mathrm{kg} \mathrm{CO} \mathrm{eq}^{\mathrm{kgh}} \mathrm{ha}^{-1}\right)\end{array}$ & $\begin{array}{c}\text { Inhibidor } \\
\text { nitrificación }\end{array}$ & $\begin{array}{c}\text { Labranza } \\
\text { mínima }\end{array}$ & $\begin{array}{c}\text { Abono } \\
\text { orgánico }\end{array}$ & $\begin{array}{c}\text { Incorporación } \\
\text { residuos }\end{array}$ \\
\hline Maíz & 1921 & 43 & 138 & 258 & 362 \\
Camote & 1802 & 48 & 122 & 226 & \\
Yuca & 1961 & 104 & 150 & 255 & \\
\hline
\end{tabular}

estimó que su uso posee un bajo potencial de reducción de la HC (3.4\%).

Las opciones individuales de mitigación modeladas y seleccionadas varían en la capacidad de reducir las HC por cultivo. Así, por la implementación de la labranza mínima en los sistemas de producción de los tres cultivos, se reflejó una reducción promedio de la HC en $7 \%$ (7.2\% en maíz, $6.8 \%$ en camote y $7.6 \%$ en yuca). Con esta práctica -coincidiendo con la literatura especializada- se ha logrado reducciones en: la compactación del suelo, riesgo de erosión, gasto energético y reducciones de hasta $19 \%$ en los costos de preparación de terreno; lo que conlleva ingresos adicionales (Benites \& Bot, 2014; Marquina et al., 2015). Con la incorporación de residuos de plantas de maíz, se reduce la $\mathrm{HC}$ en promedio $19 \%$ y al implementar todas las opciones de mitigación seleccionadas, la $\mathrm{HC}$ se reduce en $42 \%$. Para la implementación significativa de las medidas de mitigación por parte de los productores, es estratégico que las autoridades estatales del sector agrícola ejecuten las siguientes acciones: i) capacitaciones técnicas en CC, enfocadas a que la adopción de prácticas de mitigación amigables con el ambiente, se refleja en la sustentabilidad de los sistemas de producción; ii) disponer de instrumentos comerciales que promuevan una agricultura basada en bajas emisiones de GEI y que se refleje en incentivos por la conservación del ambiente y una mejor aceptación de productos alimenticios por parte de los consumidores; iii) disponer de inversiones, políticas y apoyo económico específico para los productores dispuestos a implementar medidas de mitigación.

\section{Conclusiones.}

Se comprobó que el sistema mecanizado de maíz, camote y yuca en la costa peruana son totalmente dependientes de fertilizantes sintéticos y de plaguicidas. Al cuantificar las $\mathrm{HC}$ en los tres cultivos a nivel de fincas por sitios, se obtuvo la menor $\mathrm{HC}_{\mathrm{i}}$ en la producción de yuca en Cañete con promedio de $62 \mathrm{~kg}$ $\mathrm{CO}_{2} \mathrm{eq} \cdot \mathrm{t}^{-1}$, dos veces menor a la $\mathrm{HC}_{\mathrm{i}}$ promedio de Barranca. La HC obtenida en maíz de Barranca superó hasta 1.7 veces a la estimada en Cañete. Las fincas de maíz y yuca en Barranca son consideradas altas emisores de GEI. Al considerar el transporte de cosechas, la menor y mayor HC total se obtuvo en las fincas de yuca en Cañete y Barranca. En los tres cultivos, las emisiones conjuntas por la producción y aplicación de fertilizantes aportan en promedio el $87 \%$ a la $\mathrm{HC}$ total y el resto por el uso de plaguicidas. En términos de país, la HC calculada en conjunto para maíz, camote y yuca representó el $1.47 \%$ del total de las emisiones del sector agropecuario. Se estimó el potencial de reducción de la $\mathrm{HC}$ por cultivo, por la modelación de las siguientes opciones de mitigación: labranza mínima, incorporación de residuos agrícolas, uso de abonos orgánicos procesados e inhibidores de nitrificación. Al incorporar estas cuatro opciones de mitigación se estimó reducciones promedio de GEI de hasta $42 \%$ en la producción mecanizada de maíz. Con su implementación se conforman escenarios sustentables, por cultivo, los cuales requieren de su evaluación en campo integrando el uso de la tierra y clima.

El modelo CFT se constituye en una herramienta útil y de fácil aplicación para la estimación de la $\mathrm{HC}$ en condiciones de finca, que pueden ser utilizadas por agricultores, extensionistas y tomadores de decisión.

\section{Agradecimientos.}

Agradecemos a los Programas del CGIAR sobre Raíces, Tubérculos y Bananos (RTB), Humid Tropics, Climate Change, Agriculture and Food Security (CCFAS). El autor principal agradece el apoyo brindado por la Secretaría Nacional de Ciencia y Tecnología de Panamá (SENACYT) y del Instituto de Investigación Agropecuaria de Panamá (IDIAP).

\section{Literatura citada.}

Akiyama H., Xiaoyuan Y. \& Yagi Y. 2010. Evaluation of effectiveness of enhanced-efficiency fertilizers as mitigation options for $\mathrm{N}_{2} \mathrm{O}$ and $\mathrm{NO}$ emissions from agricultural soils: meta-analysis. Global Change Biology. 16: $1837-1846$.

Andrade H., Brook R. \& Ibrahim, M. 2008. Growth production and carbon sequestration of silvopastoral systems with native timber species in the dry lowlands of Costa Rica. Plant and Soil 308(1-2): 11-22.

ASABE (American Society of Agricultural and Biological Engineers). 2006. Agricultural machinery management data. American Society of Agricultural and Biological Engineers Standard ASAE EP496.3. ASABE, St. Joseph. 385-398.

Audsley E., Stacey K., Parsons D.J. \& Williams A.G. 2009. Estimation of the greenhouse gas emissions from agricultural pesticide manufacture and use. Cranfield University. 20 p.

Benites J.R. \& Bot A. 2014. Agricultura de Conservación, una práctica innovadora con beneficios económicos y 
medioambientales. Editorial Banco Agropecuario. Lima, Perú. 344 p.

Bolaños J. 1997. Un Modelo Simplificado de la Productividad Potencial del Maíz. En: Bolaños J. (ed) Síntesis de resultados experimentales del Programa Regional de Maíz (1993-1995): 291-296. CIMMYT PRM. Guatemala.

Bouwman A.F., Boumans L.J.M. \& Batjes N.H. 2002. Modeling global annual $\mathrm{N}_{2} \mathrm{O}$ and $\mathrm{NO}$ emissions from fertilized fields. Global Biogeochem Cycles. 16(4), 1080, doi:10.1029/2001GB001812

Brentrup F. \& Pallière C. 2008. GHG emissions and energy efficiency in European nitrogen fertiliser production and use. York: The International Fertiliser Society. 639 p.

Cochran W.G. 2000. Técnicas de Muestreo, décima quinta edición. Compañía Editorial Continental S.A. México DF. 513 p.

Colomb V., Bernoux M., Bockel L., Chotte J-L., Martin S., Martin-Phipps C., Mousset J., Tinlot M. \& Touchemoulin O. 2012. Review of GHG Calculators in Agriculture and Forestry Sectors: A Guideline for Appropriate Choice and Use of Landscape Based Tools, Version 2.0 (ADEME, IRD, FAO). 43 p.

Chaparro A., Herrera O.A. \& Peña C. 2005. Consumo energético, eficiencia de campo y cobertura vegetal en labranza-siembra de maíz en el Valle del Cauca, Colombia. Acta Agronómica. 54(1): 11-17.

FAO-IFA (Organización de las Naciones Unidas para la Alimentación y la Agricultura - Asociación Internacional de la Industria de los Fertilizantes). 2004. Estimaciones globales de las emisiones gaseosas de $\mathrm{NH}_{3}, \mathrm{NO}$ y $\mathrm{NO}_{2}$ provenientes de las tierras agrícolas. Primera versión. Roma. p. 1-30.

FAO (Organización de las Naciones Unidas para la Alimentación y la Agricultura). 2015. FAOSTAT (FAO Statistics Division). Consultado 16 may. 2016. Disponible en http://faostat3.fao.org/home/E.

Feliciano D., Nayak D., Vetter S. \& Hiller J. 2015. CCAFS Mitigation Options Tool. Consultado 23 mar. 2016. Disponible en https://ccafs.cgiar.org/mitigation-optiontool-agriculture\#.V9BhUbjhC01.

Flores E.D., De la Cruz R.S.M. \& Antolin M.C. 2016. Energy Use and Greenhouse Gas Emissions of Farmer-level Sweet Potato Production Systems in the Philippines. Asian Journal of Applied Sciences. 4(1): 110-119.

Frank F., Montero G., Ricard F., Sirotiuk V. \& Viglizzo E. 2014. La huella de carbono en la agroindustria. En: Viglizzo E. (ed) Producción primaria: 27-33. Ediciones INTA, La Pampa, Argentina.

Frohmann A. \& Olmos X. 2005. Huella de carbono, exportaciones y estrategias empresariales frente al cambio climático. Comisión Económica para América Latina y el Caribe (CEPAL). Agencia Española de Cooperación Internacional para el Desarrollo (AECID). $76 \mathrm{p}$.

Gardi C., Angelini M., Barceló S., Comerma, J., Cruz Gaistardo C., Encina Rojas A., Jones A., Krasilnikov P., Mendonça Santos M.L., Montanarella L., Muñiz Ugarte O., Schad P., Vara Rodríguez M.I. \& Vargas R. 2014. Atlas de suelos de América Latina y el Caribe, Comisión Europea-Oficina de Publicaciones de la Unión Europea, L-2995 Luxembourg. 176 p.
Haverkort A.J. \& Hillier J.G. 2011. Cool Farm Tool - Potato: Model Description and Performance of Four Production Systems. Potato Research. 54: 355-369.

Herrero M., Havlík P., Valin H., Notenbaert A., Rufino M.C., Thornton P.K., Blümmel M., Weiss F., Grace D. \& Obersteiner M. 2013. Biomass use, production, feed efficiencies, and greenhouse gas emissions from global livestock systems. Proc Natl Acad Sci USA. 110(52): 2088-2093.

IFA (Asociación Internacional de la Industria de los Fertilizantes, Italia). 2009. Fertilizers, climate change and enhancing agricultural productivity sustainably, primera edición. International Fertilizer Industry Association. Paris, France. 30 p.

IPCC (Intergovernmental Panel on Climate Change). 2007. Climate Change 2007, Impacts adaptation and vulnerability. En: Parry, ML; Canziani, OF; Palutikof, JP; van der Linden, PJ; Hanson, CE (eds) Contribution of working group II to the fourth assessment report of the Intergovernmental Panel on Climate Change. Cambridge UK: Cambridge University Press. p. 976-2007.

IPCC (Intergovernmental Panel on Climate Change). 2013. Climate Change 2013: The Physical Science Basis. En: Stocker T.F., Qin D., Plattner G.K., Tignor M., Allen S.K., Boschung J., Nauels A., Xia Y., Bex V. \& Midgley P.M. (eds) Contribution of Working Group I to the Fifth Assessment Report of the Intergovernmental Panel on Climate Change: 976-2007. Cambridge University Press. Cambridge UK.

Lal R. 2004. Carbon emissions from farm operations. Environ Int. 30: 981-990.

Linquist B.A., Adviento-Borbe M.A., Pittelkow C.M., van Kessel C. \& van Groenigen K.J. 2012. Fertiliser management practices and greenhouse gas emissions from rice systems: A quantitative review and analysis. Field Crops Research 135: 10-21.

Ma B.L., Liang B.C., Biswas D.K., Morrison M.J. \& McLaughlin N.B. 2014. The carbon footprint of maize production as affected by nitrogen fertilizer and maizelegume rotations. Nutrient Cycling in Agroecosystems. 94(1): 15-31.

Marquina S., Pérez T., Donoso L., Giulante A., Rasse R. \& Herrera F. 2015. NO, $\mathrm{N}_{2} \mathrm{O}$ and $\mathrm{CO}_{2}$ soil emissions from Venezuelan corn fields under tillage and no-tillage agriculture. Nutr Cycl Agroecosyst. 101: 123-137.

Milne L., Neufeldt H., Rosenstock T., Smalligan M., Cerri C.E., Malin D., Easter M., Bernoux M., Ogle S., Casarim F., Pearson T., Bird D.N., Steglich E., Ostwald M., Denef K. \& Paustian K. 2013. Methods for the quantification of GHG emissions at the landscape level for developing countries in smallholder. Environmental Research Letters 8.9 p.

MINAGRI (Ministerio de Agricultura y Riego). 2015. Sistema Integrado de Estadística Agraria. Insumos y Servicios Agrarios. Estadística de insumos agrarios. Lima, Perú. pp. 138-141.

Montaldo A. 1991. Cultivo de raíces y tubérculos, segunda edición. Instituto Interamericano de Cooperación para la Agricultura (IICA). Libros y materiales educativos. San José, Costa Rica. No. 21. 408 p.

Moran D., MacLeod M., Wall E., Eory V., McVittie A., Barnes A., Rees B., Pajot G., Matthews R., Smith P. \& Moxey A. 2008. Developing carbon budgets for UK 
agriculture, land-use, land-use change and forestry out to 2022. Climate Change 105: 529-553.

Nayak D., Saetnan E., Cheng K., Wang W., Koslowski F., Cheng Yan-Fen., Yun Zhu W., Wang Jia-Kun., Liu JianXin. \& Moran D. 2015. Management opportunities to mitigate greenhouse gas emissions from Chinese agricultura. Agriculture, Ecosystems \& Environment 209: 108-124.

Nyakatawa E.Z., Mays D.A., Way T.R., Watts D.B., Torbert H.A. \& Smith D.R. 2011. Tillage and Fertilizer Management Effects on Soil-Atmospheric Exchanges of Methane and Nitrous Oxide in a Corn Production System. Applied and Environmental Soil Science. 12 p.

Ogle S.M., Breidt F.J. \& Paustian K. 2005. Agricultural management impacts on soil organic carbon storage under moist and dry climatic conditions of temperature and tropical regions. Biogeochemistry. 72: 87-121.

Olander L.P. 2011. Using Biogeochemical Process Models to Quantify Greenhouse Gas Mitigation from Agricultural Management Projects. Nicholas Institute for Environmental Policy Solutions. 35 p.

Olivet Y., Ortiz A., Cobas D., Blanco A. \& Herrera E. 2012. Evaluación de la labranza para el cultivo de boniato (Ipomea batatas L.) en un suelo Fluvisol. Re. Cie. Agr. 21: 24-29.

Pandey S. \& Gardner C.O. 1992. Recurrent selection population, variety and hybrid improvement in tropical maize. Adv. Agron. 48: 1-87.

Richards M., Metzel R., Chirinda N., Ly P., Nyamadzawo G., Duong Q., de Neergaard A., Oelofse M., Wollenberg E., Keller E., Malin D., Olesen J.E., Hillier J. \& Rosenstock T.S. 2016. Limits of agricultural greenhouse gas calculators to predict soil $\mathrm{N}_{2} \mathrm{O}$ and $\mathrm{CH}_{4}$ fluxes in tropical agriculture. Sci. Rep. 6: 1-8.

Rosenzweig C., Jones J.W., Hatfield J.L., Ruane A.C., Boote K.J., Thorburn P., Antle J.M., Nelson G.C., Porter C., Janssen S., Asseng S., Basso B., Ewert F., Wallach D., Baigorria G. \& Winter J.M. 2013. The agricultural model intercomparison and improvement project (AgMIP): protocols and pilot studies. Agricultural and Forest Meteorology. 170: 166-182.

SEEG (Sistema de Estimación de Emisiones de Gases de Efecto Invernadero, Perú). 2014. Sector agropecuario (1990-2013). Perú. Consultado 03 jun. 2016. Disponible en http://seegperu.org.

Shan J. \& Yan X. 2013. Effects of crop residue returning on nitrous oxide emissions in agricultural soils. Atmospheric Environment. 71: 170-175.

Smith P., Martino D., Cai Z., Gwary D., Janzen H., Humar P., McCarl B., Ogle S., O’Mara F., Rice C., Scholes B., Sirotenko O., Howden M., McAllister T., Pan G., Romanenkov V., Schneider U. \& Towprayoon S. 2007. Policy and technological constraints to implementation of greenhouse gas mitigation options in agriculture. Agriculture, Ecosystems \& Environment. 118: 6-28.

van Kessel C., Venterea R., Six J., Adviento-Borbe M.A., Linquist B. \& van Groenigen K.J. 2013. Climate, duration, and $\mathrm{N}$ placement determine $\mathrm{N}_{2} \mathrm{O}$ emissions in reduced tillage systems: a meta-analysis. Global Change Biology. 19(1): 33-44.

WB (World Bank). 2013. $\mathrm{CO}_{2}$ emissions (metric tons per capita). Carbon Dioxide Information Analysis Center, Environmental Sciences Division, Oak Ridge National Laboratory, Tennessee, USA. Consultado 09 jun. 2016. Disponible http://data.worldbank.org/indicator/EN.ATM.CO2E.PC.

Wolf J. 2002. Comparison of two potato simulation models under climate change. II. Application of climate change scenarios. Climate Research. 21: 187-198.

World Resources Institute. 2014. CAIT Climate data explorer. Consultado 13 ene. 2016. Disponible en http://cait.wri.org/.

Zhang Wei-feng., Dou Zheng-xia., He P., Ju Xiao-Tang., Powlson D., Chadwick D., Norse D., Lu Yue-Lai., Zhang Y., Wu L., Chen Xin-Ping., Cassman K.G. \& Zhang FuSuo. 2013. New technologies reduce greenhouse gas emissions from nitrogenous fertilizer in China. Proc Natl Acad Sci USA. 110(21): 8375-8380.

1 Graduando del Programa de Doctorado en Agricultura Sustentable. Universidad Nacional Agraria La Molina. Lima, Perú. Instituto de Investigación Agropecuario de Panamá (IDIAP). International Potato Center (CIP). Crop and Systems Sciences Division. Lima, Perú. rodrigoamoralesa@ gmail.com.

2 Universidad Nacional Agraria La Molina. Facultad Agronomía. Lima, Perú. percyz@lamolina.edu.pe. Autor de correspondencia.

3 Universidad Nacional Agraria La Molina. Facultad de Economía y Planificación. Lima, Perú. fmendiburu@lamolina.edu.pe. International Potato Center (CIP). Crop and Systems Sciences Division. Lima, Perú. f.mendiburu@cgiar.org.

4 International Potato Center (CIP). Crop and Systems Sciences Division. Lima, Perú. r.quiroz@cgiar.org. 